\title{
INTEGRAÇÃO ENSINO-TRABALHO-CIDADANIA NA FORMAÇÃO DE ENFERMEIROS
}

\author{
Suzelaine TAN JIa, Carmen M aria dos Santos Lopes M onteiro Dantas da SI LVAa, \\ Verônica Santos A L BU QU E RQU E ${ }^{b}$, Lígia de Oliveira VIANAc ${ }^{c}$ N eiva M aria Picinini dos SANT OSc
}

\section{RESUMO}

0 presente estudo tem como objetivo identificar os conflitos encontrados no cotidiano do trabal ho no decorrer do curso de especial ização Processo de M udança no Ensino Superior e nos Serviç̧os de Saúde. E studo qualitativo descritivo, tipo estudo de caso; o cenário foi um centro universitário privado situado no estado do Rio de Janeiro. Os sujeitos foram 18 estudantes do curso de especialização, que se dispuseram espontaneamente a participar do estudo. A coleta de dados ocorreu em abril de 2009, os dados foram analisados e descritos em cinco unidades temáticas que descrevem os focos de conflitos no mundo do trabal ho: A integração ensino, trabal ho e cidadania; 0 trabalho em equipe; A educação permanente; A responsabilidade social; 0 estabelecimento da linha de cuidado. A interseção entre a seara acadêmica e os serviços de saúde produz tensões e conflitos, que na maioria das vezes estão latentes, mas não manifestos.

Descritores: Ensino. Formação de recursos humanos. E ducação em enfermagem.

\section{RESUMEN}

E I actual estudio quetiene como objetivo identificar los conflictos encontrados dentro del trabajo cotidiano en el transcurso del curso de especialización "E I proceso del cambio en la educación superior y los servicios de la salud". E I estudio cualitativo descriptivo, tipo estudio de caso, el escenario fue un centro privado universitario en el estado de R io de J aneiro, B rasil. L os ciudadanos fueron 18 estudiantes del curso deespecialización, que sedispusieron espontáneamentea participar del estudio. L a recogida de datos ocur rió en abril de 2009, los datos fuer on analizados y descritos en cinco unidades temáticas que describen los focos de conflictos en el mundo del trabajo: E I trabajo y la ciudadanía de la educación de la integración; E I trabajo en equi po; $L$ a educación permanente; $L$ a responsabilidad social; $E$ I establ ecimiento dela línea del cuidado. $L$ a intersección entre el ter reno del académico y los servicios médicos producen tensiones y conflictos, que la mayor parte del tiempo están latentes, pero no se manifiestan.

Descriptores: E nseñanza. E ducación en enfer mería. F or mación de recursos humanos. Título:I ntegración enseñanza-trabajo-ciudadanía en el oficio de enfer mera.

\section{ABSTRACT}

This study aims to identify the conflicts found daily throughout the development of the specialization course P rocess of Changein Superior $\mathrm{E}$ ducation and $\mathrm{H}$ ealth Services. This descriptive qual itativestudy is a casestudy car ried out at a private U niversity Center in the State of R io de J aneiro, B razil. T he subjects w ere 18 students from the specialization course, who spontaneously decided to participate in the study. The data was collected in A pril 2009 and analyz ed and described in five thematic units that describethefocus of conflicts in thew orld of thew ork: Integration among $E$ ducation, W ork and Citiz enship; T eam w ork; P ermanent education; Social responsibility; E stabl ishment of the careline T heinter section betw een the academic field and health services produces tensions and conflicts which are latent most of the time, but are never evident.

Descriptors: T eaching. E ducation, nursing. H uman resources formation.

Title:Integrating teaching, nursing work and citizenship.

\footnotetext{
a Enfermeira, D outoranda da Escola de Enfermagem Anna Nery (EEAN) da U niversidade Federal do Rio de Janeiro (UFRJ), D ocente do Curso de Graduação em Enfermagem do Centro U niversitário Serra dos Órgãos, T eresópolis, Rio de Janeiro, Brasil.

${ }^{b}$ D outora em Ciências, D ocente do Curso de Graduação em Enfermagem do Centro U niversitário Serra dos Órgãos, Teresópolis, Rio de Janeiro, Brasil.

'D outora em Enfer magem, Professora A djunta da EEAN / U F RJ, D ocente dos Cursos de G raduação em Enfermagem e de Pós-G raduação em Enfer magem Stricto Sensu da EEAN / U F RJ, Rio de Janeiro, Brasil.
} 


\section{INT RODUÇÃO}

A formação e o trabalho dos profissionais de saúde na América Latina vêm sendo decisivamente impactados pela reorganização dos sistemas de saúde, pelas pressões para a reforma da U niversidade e pelo processo de descentralização políticoadministrativa do Estado. As iniciativas comprometidas com a relevância social da universidade e dos processos de formação no campo da saúde têm historicamente procurado articular dois contextos, aparentemente desconectados - universidades e serviços - buscando ligar os espaços de formação aos diferentes cenários da vida real e de produção de cuidados à saúde.

Considerando a experiência acumulada nos últimos cinqüenta anos, particularmente no campo da educação médica, e reconhecendo a inadequação dos modelos de formação para enfrentar os desafios atuais da atenção à saúde, é formado no início dos anos noventa, por iniciativa da F undação Kellogg, o Programa U ma N ova I niciativa na E ducação dos Profissionais de Saúde - U nião com a Comunidade (UNI). Sua proposição central se baseou na relação de parceria entre a universidade, os serviços locais de saúde e a comunidade, como 0 alicerce sobre 0 qual devem estar fundados os processos de transformação da educação dos profissionais e dos sistemas de saúde. A té então, esses atores estabeleciam entre si relações bilaterais, expressas pela Integração DocenteAssistencial (U niversidade-Serviços), pela Extensão U niversitária (U niversidade-Comunidade) e pela Atenção Primária à Saúde (Serviços-Comunidade). $O$ UNI propõe, em contraposição, articular esses três atores num sistema mais complexo e orientado para inovação das práticas de saúde e da for mação profissional ${ }^{(1)}$.

N este contexto, o encontro entre a formação dos profissionais da saúde e a produção dos cuidados em saúde vem passando ao longo das últimas décadas por alterações conceituais importantes: de Integração D ocente-Assistencial (IDA) à Integração Ensino-Serviço (IES) ou integração EnsinoServiço-Comunidade (IESC) até a Integração E nsino-T rabal ho-Cidadania (IET C).

Existem diferenças importantes entre a relação bilateral concebida na Integração D ocenteA ssistencial (IDA) e a Articulação T ripla E nsinoServiço-Comunidade (IESC): a IDA contempla o ensino e a pesquisa pelo lado docente e a atenção à saúde pelo âmbito assistencial. Já a IESC, além dos aspectos considerados na IDA, amplia seu campo de atuação, incorporando a participação popu$\operatorname{lar}^{(2)}$.

De integração Ensino-Serviço-Comunidade (IESC) para Integração Ensino-T rabalho-Cidadania (IETC) operam-se novas ampliações significativas: trocar a palavra serviço por trabalho tem por base a concepção que não se produz cuidados apenas em serviços de saúde. A abrangência de atuação é ampliada para qualquer espaço de produção direta ou indireta da vida, onde haja espaço para atuação dos profissionais de saúde. A substituição de comunidade por cidadania, também opera uma ampliação - além da participação popular, começa a se contemplar o controle social, especialmente no contexto do Sistema Ú nico de Saúde. Cabe ressaltar, ainda, que a IETC, no âmbito do ensino, incorpora o protagonismo estudantil, além da docência e da pesquisa.

Para tanto o objeto investigativo do estudo se relaciona ao impacto provocado pelo curso de especialização em processos de mudança, com foco nas questões que envolvem a integração ensinotrabalho-cidadania.

0 objetivo da presente pesquisa foi identificar os conflitos encontrados no cotidiano do trabalho no decorrer do curso de especialização do processo de mudança no ensino superior e nos serviços de saúde.

\section{METODOLOGIA}

T rata-se de um estudo qualitativo descritivo, tipo estudo de caso, cujo cenário foi um Centro U niversitário Privado situado no Estado do Rio de Janeiro. Os sujeitos foram os estudantes do curso de especialização em Processos de M udança no E nsino Superior e nos Serviços de Saúde, que estavam regularmente matriculados e se dispuseram a participar do estudo. Foram encaminhados setenta e quatro instrumento de coleta de dados, juntamente com o termo de consentimento livre esclarecido e uma carta convite a participar do estudo, entretanto retornaram apenas dezoito, esse foram os selecionados para compor o grupo de sujeitos do estudo e para preservar 0 anonimato foram identificados de (E 1) a (E 18).

Os participantes foram informados sobre a pesquisa, a importância do desenvolvimento do estudo, levando em consideração os aspectos éti- 
cos e o rigor científico de acordo com o que determina a Resolução no 196/ 96 do Conselho Nacional de Saúde ${ }^{(3)}$.

A coleta de dados ocorreu em abril de 2009, através de um instrumento com perguntas abertas, após aprovação do Comitê de Ética de Pesquisa (no 342/09). 0 tratamento dos dados fundamentou-se no método análise temática, realizada em três momentos distintos: inicialmente o momento da organização dos dados coletados; seguidamente da transcrição das entrevistas, permitindo eleger as unidades temáticas; e por fim o momento do tratamento dos resultados com a inferência e a interpretação, na perspectiva de validar as unidades elaboradas na fase anterior.

\section{RESULTADOS E DISCUSSÃO}

Para subsidiar a análise dos resultados encontrados no presente estudo, cabe-nos iniciar apresentando brevemente o referencial teórico sobre integração ensino-trabalho-cidadania (IETC) utilizado.

Os resultados foram cuidadosamente submetidos à análise e descritos em unidades temáticas para melhor apreciação do processo investigativo que objetivou o estudo, bem como pela necessidade de atender à metodologia proposta. Desta forma, apresentamos a questão geradora do processo investigativo de modo a identificar os conflitos encontrados no cotidiano do trabal ho no decorrer do curso de especialização do processo de mudança no ensino superior e nos serviços de saúde, e, por conseguinte originaram cinco unidades temáticas: A integração Ensino T rabalho e Cidadania como foco de conflitos no mundo do trabal ho; 0 trabalho em equipe como foco de conflitos no mundo do trabalho; A educação permanente como foco de conflitos no mundo do trabal ho; A responsabilidade social como foco de conflitos no mundo do trabalho; 0 estabelecimento da linha de cuidado como foco de conflitos no mundo do trabalho. A unidade temática com maior representatividade das respostas, os quais os participantes pactuam do mesmo pensar, estão ordenadas, conforme relacionadas acima, de forma decrescente.

A integração Ensino Trabalho e Cidadania (IETC) como foco de conflitos no mundo do trabalho: durantes os doze meses de realização do curso de especialização em Processos de Mudan- ça no Ensino Superior e nos Serviços de Saúde, o IET C foi um tema amplamente discutido, o que na mudança curricular dos cursos de enfermagem, medicina e odontologia, representa a integração dos estudantes no cenário prático.

Outrossim, a situação problema que gerou esta reflexão, em seu teor/ contexto continham elementos agregadores e disparadores das seguintes questões: a refletir sobre a centralidade da interlocução entre o ensino e o mundo do trabalho nas propostas de mudança na formação dos profissionais da saúde; abordar os cenários de aprendizagem, incluindo sua diversificação, o papel docente e discente na inserção no mundo do trabaIho e os pressupostos da educação permanente; abranger as relações político-institucionais no movimento de integração ensino-trabalho-cidadania(4).

Pela abrangência destas questões supracitadas e pela caracterização que este cenário de aprendizagem tem significado na mudança curricular do curso de graduação em enfermagem, medicina e odontologia, é salutar que conflitos apareçam para que evidenciem os possíveis pontos de divergências e convergências entre o mundo do trabalho e do ensino. $E$ em virtude destas, o grupo de especializando e docentes facilitadores do processo de aprendizagem, encaminharam pela vias do diálogo e discussão, tais questões a fim de buscar alternativas de melhorias à qualidade do ensino e assistência oferecida aos usuários do sistema de saúde, pois são eles próprios que estão vivenciando o processo.

Outro ponto a destacar é que, os estudantes estão sendo inseridos nestes ambientes de aprendizagem, conscientes de que são ou farão parte integrante da equipe de trabalho ou da comunidade. Por esta razão, que a integração ensino-trabal hocidadania, deve tentar gradativamente mediar a articulação processual de ensino-aprendizagem, da orientação teórica através das práticas assistenciais em consonância com o nível individual e coletivo(5). $^{(5)}$.

Há que se considerar ainda, que o cenário de aprendizagem não deve se limitar em determinados locais de desenvolvimento de práticas profissionais como espaços físicos de trabalho-tarefas, mas sim, de representar espaços em que as relações inter e intrapessoais de envolvimentos dos sujeitos sejam eficazmente desenvolvidas, que possibilitem a inclusão do estudante ao processo 
de produção de serviço. Enfim, que possam provocar mudanças no processo de formação profissional do estudante e na comunidade atendida, obtendo-se o bem estar para todos ${ }^{(6)}$. Assim os cenários de aprendizagem podem ter como um dos objetivos fundamentais, o desenvolvimento do processo ensino-aprendizagem na realidade social dos serviços de saúde e da comunidade ${ }^{(7)}$, pois, entendemo-los repercutindo sempre na Escola (U niversidade).

Segue alguns depoimentos dos participantes que congregaram a esta unidade temática:

A integração dos alunos ao ambiente da unidade de saúde fica somente na responsabilidade do professor. As equipes que estão no serviço não recebem as informaçôees necessárias para entender o processo ensino a prendizagem no novo currículo isso gera divergência e atitudes desfavoráveis ao ensino (E 13).

P orque penso que o link ensino-serviço ainda não é muito efetivo (E5).

Inserir 0 aprendizado à prática no serviço em favor ao cidadão não éuma realidade (E 4).

Essas falas acima vêm a comprovar a necessidade de aproximação desses atores do mundo do trabalho e do ensino, e demonstram a existência de uma lacuna e um elo de comunicação entre esses dois univer sos tão complexos, que nem sempre se dialogam de modo harmonioso. Até, porque de acordo com a experiência da prática laboral em saúde, vivemos velozes integrados em contextos sociais tão dinâmicos, que se não formos devidamente preparados e fortes diante das mudanças que se impõem os cercos humanos, nos quais dizemos estar em serviço para com o outro, tenderão a perecer. Desta forma é preciso pensar-se em alter nativas para o trabal ho interdisciplinar, encontrando a interface ideal das profissões ${ }^{(8)}$.

Os atores envolvidos devem estar revisitando a integração denominada como Ensino T rabaIho e Cidadania, pois é basilar para o verdadeiro transformar perpassando-a ativamente pelos profissionais que se formam, mas, sem nunca desprezar aqueles que já se encontram no exercício das atividades propriamente ditas. Para tal, precisamos constantemente rever o cotidiano nas enraizadas convicções, que tantas vezes petrificam congelando 0 social, tornando-nos opositores às inovações e adversos a tantos atores principalmente se estes trilham ainda os meandros do aprender a conhecer pelo fazer como se perfilam os estudantes que estão caminhando pela formação.

De acordo com as idéias apresentadas, tais sentidos ficam mais claros se puderem ser explicados do seguinte modo: "a graduação dura somente alguns anos, enquanto a atividade profissional pode permanecer por décadas e que os conhecimentos e competências vão-se transformando velozmente, torna-se essencial pensar em uma metodologia para uma prática de educação libertadora, na formação de um profissional ativo e apto a aprender a aprender"(9)

Todavia, o curso de especialização por ser formado por estudantes desses dois universos, e por agregar componentes que supostamente estão dispostos a provocarem mudanças, esses conflitos se torna elementos ativadores e instigadores da curiosidade na ampliação do conhecimento nesta área. D este modo, fazendo com que esses estudantes avidamente busquem outras experiências neste sentido para fortalecer ainda mais a mudança curricular proposta para os cursos de graduação.

M uito embora, não é possível pensar a mudança na formação dos profissionais de saúde sem a discussão sobre a articulação ensino-trabalho, considerando-a um espaço privilegiado para uma reflexão sobre a realidade da produção de cuidados e a necessidade de transformação do modelo assistencial vigente em um modelo que considere como objetivo central as necessidades dos usuá$\operatorname{rios}^{(5)}$

0 trabalho em equipe como foco de conflitos no mundo do trabalho: temas como a interdisciplinaridade, transver salidade, equidade e multidisciplinaridade foram questões traçadas como objetivo a ser atingido pelos estudantes e, portanto, exaustivamente discutidas na produção do conhecimento deste curso de especialização. A destacar ainda que a situação problema que ger ou esta reflexão, em seu teor/ contexto continham elementos agregadores e disparadores das seguintes questões: refletir sobre o trabalho em equipe de saúde e suas possibilidades desde a formação, incluindo sua potência formativa, de produção de coletivo, de integralidade e de democratização das relações de trabalho e a estimular a discussão sobre responsabilidade profissional, orquestração do trabalho em saúde, competência coletiva e atos corporativos ${ }^{(4)}$. 
Os profissionais da área da saúde não vivenciam, ao longo de sua formação, estratégias que articulem suas atividades e saberes com as dos outros profissionais da equipe. Sempre é referido como um dos momentos de dificuldade, quando se iniciam as atividades profissionais, 0 aprendizado em que se torna necessário compartilhar com outros profissionais os espaços de atuação e os sujeitos daquela atuação. 0 aprendizado gera, muitas vezes, situações extremamente conflituosas nas quais acabam prevalecendo as vaidades individuais em detrimento de um cuidado mais qualificado. Diante desse tipo de conflito, destacamos que se torna ainda mais significativo colocar o usuário no centro do debate, pois assim é possível evocar os valores éticos que servem de justificativa última para a atuação de todos os profissionais de saúde, criando um terreno comum para o diálo$\mathrm{go}^{(5)}$.

Essas questões acima apresentadas podem observar nos depoimentos dos participantes que seguem:

0 discurso é de interdisciplinaridade e multidisciplinaridade, desde épocas remotas. $M$ as, não éo que estamos vendo ainda na prática. É necessário mudar urgente (E 8).

As pessoas ainda jogam bastante com poderes e vaidades, bem como grande dificuldade de trabal har em equipe e constr uir o consenso (E 11).

A falta de integração e respeito em relação a alguns colegas [ ...] temos uma relação de multidisciplinaridade, porém não inter-profissional, mas acredito que isto possa ser mudado com paciência e vontade [ ...] (E 14).

O bservo ainda um distanciamento de muitos profissionais [ ...] (E 16).

Excesso de tar efas e alunos e pouco tempo par exercer um trabal ho em equipe [...] (E 7).

E mbora, sejam inquietantes tais situações precisamos tratá-las com delicadeza porque se permeiam de dúvidas e inseguranças para os sujeitos implicados, em que a combinação de habilidades, para o trabalho em equipe (re)desenha 0 desequilíbrio e os desencontros contemporâneos em matéria de recursos humanos em saúde ${ }^{(10)}$. A pesar disso, como propostas de construção destes desafios na formação dos enfermeiros estão práticas pedagógicas inovadoras, que apontam a co-responsabilidade dos setores da saúde e educação com base em modelos de ensino integral e interdisciplinar, para um estudante que almeja significar as necessidades prementes de saúde e a autogestão do aprender ${ }^{(11)}$.

A educação permanente como foco de conflitos no mundo do trabalho: os pressupostos da educação permanente foram trabalhados neste curso de especialização como um tema transversal, onde várias situações problemas encaminhavam para esta reflexão/ discussão.

A tualmente, não é possível pensar na interface entre ensino e trabal ho sem remeter-se - declinar-se à educação permanente. $N$ este sentido, é preciso contextualizar a integração ensino-trabaIho, neste movimento sincronizado: profissionais de saúde, docentes e estudantes devem estar inseridos nas estratégias de educação permanente, tendo em vista melhorar a formação e fortalecer - Sistema Ú nico de Saúde(5).

E m face ao supracitado, apresentamos os depoimentos dos participantes:

Há necessidade no meu cenário de prática de discutir as intercessões entre os difer entes profissionais (E 3).

Acredito que com esta metodologia todos os aspectos relacionados afins, ficam evidentes em conflito, pois ge ram constantes reuniões na tentativa de melhoria desses cenários (E 6).

Desta forma, é importante destacar que o “M inistério da Saúde está propondo a educação permanente como estratégia de transformação das práticas de formação, de atenção, de gestão, de formulação de políticas, de participação popular e de controle social no setor da Saúde. Possibilidade de transformar as práticas profissionais existe porque perguntas e respostas são construídas a partir da reflexão de trabalhadores e estudantes sobre o trabal ho que realizam ou para o qual se preparam. A educação permanente pode ser entendida como aprendizagem-trabalho, ou seja, ela acontece no cotidiano das pessoas e das organizações. Ela é feita a partir dos problemas enfrentados na realidade e leva em consider ação os conhecimentos e as experiências que as pessoas já têm" ${ }^{\prime \prime 2}$

Há que se considerar que a proposta da educação permanente parte de um desafio central: a formação e o desenvolvimento devem ocorrer de 
modo descentralizado, ascendente e transdisciplinar, ou seja, em todos os locais, envolvendo vários saberes, e com isso o resultado esperado é a democratização dos espaços de trabalho, a ampliação e incentivo da capacidade de aprender e de ensinar de todos os atores envolvidos, a busca de alter nativas criativas para os problemas encontrados, o trabalho em equipe matricial, a meIhoria per manente da qualidade do cuidado à saúde e a humanização do atendimento ${ }^{(12)}$.

Sabe-se também que a educação permanente se envolve na aprendizagem moldada pelos significados que ao reproduzirem multiplicando sentidos, têm como matriz o revitalizar das práticas profissionais revertendo na rede de serviços existentes nos ambientes de cuidado em prol da rede de serviços e dos usuários. Assim, têm-se como alimento os processos de capacitação originários da contextualização dos espaços de trabalho objetivando-se: "[ ...] a transformação das práticas profissionais e da própria organização do trabaIho, tomando como referência as necessidades de saúde das pessoas e das populações, da gestão setorial e do controle social em saúde"(10).

A responsabilidade social como foco de conflitos no mundo do trabalho: 0 setor da Saúde é responsável pela maior política brasileira de inclusão social. O Sistema Ú nico de Saúde (SUS), criado para atender a todos os cidadãos, é considerado a mais importante reforma de Estado em curso no País, todavia, o fortalecimento do SUS, depende diretamente de pessoas dos diversos segmentos sociais, pessoas que têm a tarefa ética e política de dar seguimento ao processo iniciado pelo M ovimento Sanitário(12).

Outrossim, a idéia é que, a partir de agora, os processos de qualificação dos trabalhadores da saúde sejam orientados pelas necessidades de saúde da população, do próprio setor da Saúde e do controle social, ou seja, eles devem responder a indagações como: 0 que é ou quais são os problemas que afastam nossa prática da atenção integral à saúde e de qualidade? Por quê? Como mudar essa situação? A educação deve servir para preencher lacunas e transformar as práticas profissionais e a própria organização do trabalho(12).

Para tanto, não basta apenas transmitir novos conhecimentos para os profissionais, pois 0 acúmulo de saberes técnico é apenas um dos aspectos para a transformação das práticas e não 0 seu foco central. A formação e o desenvolvimen- to dos trabalhadores também têm que envolver os aspectos pessoais, os valores e as idéias que cada profissional tem sobre o SU S. Na proposta da educação permanente, a capacitação da equipe, os conteúdos dos cursos e as tecnologias a serem utilizadas devem ser determinados a partir da observação dos problemas que ocorrem no dia-a-dia do trabalho e que precisam ser solucionados para que os serviços prestados ganhem qualidade, e os usuários fiquem satisfeitos com a atenção prestada(12).

A credito que o maior compromisso que devemos ter em qualquer cenário que atuamos é 0 de responsabilizarmos socialmente e abraçarmos a causa da necessidade das transformações sociais (E 1).

A responsabilidade social como foco de conflitos no mundo do trabalho, foi um item bastante trabal hado no curso de especialização, quando se busca refletir sobre a responsabilidade presente e futura com a existência e com as condições e a qualidade de vida dos indivíduos, da sociedade e de toda a biosfera.

0 estabelecimento da linha de cuidado como foco de conflitos no mundo do trabalho: também como todas as outras unidades temáticas, apesar da ínfima parte dos depoentes estarem em concordâncias sobre esta questão, não a faz menos importante no contexto geral do estudo, sendo que foi discutido com a mesma intensidade que as demais unidades temáticas dentro do conteúdo temático do curso de especialização, cujo objetivo foi de propiciar reflexões sobre as concepções de cuidado; examinar a relação do cuidado com as práticas terapêuticas, com as práticas populares e com os espaços públicos e privados de saúde; incluir a reflexão sobre os fundamentos da solidariedade na formação e na atenção à saúde; buscar fomentar a discussão sobre a relação do autocuidado, do cuidado com o outro e com o planeta, considerando como ponto central a relação homemnatureza; e refletir sobre a pertinência de discutir ecologia na formação dos profissionais da área de saúde a partir da essencialidade da relação entre os seres humanos e a natureza e a partir do conceito histórico de ambiente, construído pela ação humana.

A presentamos a seguir um depoimento que caracterizou esta unidade temática:

É difícil inserir [ garantir] a continuidadedo cuidado ao usuário após a alta hospitalar (E 2). 
Há no agir em matéria de cuidado noções bem definidas de humanização no exercício de formação profissional quando nos confrontamos com 0 poder da educação, sempre que esta interage com o social/ meio/ espaço de cuidado, convivendo-se com 0 que nos ultrapassa suprimindo 0 que nos limita, em que o processo de mudança age comandado pelos indivíduos.

\section{CONSIDERAÇÕES FINAIS}

A integração ensino-trabalho é um ideal a ser perseguido quando se propõe construir um currículo integrado, onde teoria e prática precisam ser indissociáveis. Tal integração não é tarefa fácil, como se observou nos relatos apresentados neste e em outros estudos nacionais.

A interseção entre a seara da acadêmica e os serviços de saúde produz tensões e conflitos, que na maioria das vezes estão latentes, mas não manifestos. Entendemos que propiciar espaços para reflexão conjunta (entre atores do ensino e atores dos serviços) é fundamental para gerir estes conflitos, a começar por (re)conhecer o trabalho do outro e identificar objetivos em comum. $\mathrm{Na}$ nossa experiência, o Curso de Especialização em Processos de M udança no Ensino e nos Serviços de Saúde foi capaz de proporcionar tais espaços de encontro, problematização, reconhecimento e alteridade.

Assim sendo, os conflitos apresentados se colocam, então, como diagnósticos para intervenção e aproximação em prol da efetiva integração ensino-trabal ho-cidadania.

\section{REFERÊNCIAS}

1 Almeida M , Feuerwerker L, Llanos M, organizadores. A educação dos profissionais de saúde na A mérica Latina: teoria e prática de um movimento de mudança. São Paul o: H ucitec; Buenos A ires: Lugar; L ondrina: Ed. UEL; 1999.

2 Ceccim RB. Integração ensino-trabalho-cidadania: responsabilidade social na educação superior. In: A nais da Semana de Responsabilidade Social do U N IF E SO; 2009 out 19-25; Teresópolis, Brasil. Teresópol is: U N IFESO; 2009. p. 19-25.

3 M inistério da Saúde (BR), Conselho $N$ acional de Saúde. Resolução 196, de 10 de outubro de 1996: diretrizes e normas regulamentadoras de pesquisa envolvendo seres humanos [ Internet] . Brasília (D F ); 1996 [ citado 2010 jun 27]. Disponível em: http:/ / conselho.saude.gov.br/ resolucoes/ reso_96.htm.

4 Centro U niversitário Serra dos Órgãos. Caderno de situações problemas. In: M oço ESM, M iranda JFA, Batista RS, Albuquerque VS. Curso Especialização em Processos de M udança no Ensino Superior e nos Serviços de Saúde. Teresópolis; 2008. p. 4-7.

5 A lbuquerque VS, Gomes AP, Rezende CH A, Sampaio MX, D ias OV, L ugarinho RM . A integração ensinoserviço no contexto dos processos de mudança na formação superior dos profissionais da saúde. Rev Bras E duc M ed. 2008;32(3):356-62.

6 T anji S, Silva CM SL M D, Viana LO, Santos N M P. Os cenários de aprendizagens na produção do conhecimento em enfermagem. Rev Enferm UFPE On L ine [ I nternet] . 2009 [ citado 2009 jun 20];3(3):1606. D isponível em: http:/ / www.ufpe.br/ revista enfermagem/ index.php/ revista/ article/ view/ 169/ 169.

7 Feuerwerker L, Costa H, Rangel M L. Diversificação de cenários de ensino e trabalho sobre necessidades/ problemas da comunidade. Divulg Saúde Debate. 2000;22:18-24.

8 Roese A, Souza AC, Porto GB, Colomé ICS, Costa LED. A produção do conhecimento na enfermagem: desafios na busca de reconhecimento no campo interdisciplinar. Rev G aúcha Enferm. 2005;26(3): 302-7.

9 M itre SM, Siqueira-Batista R, G irardi-M endonça $J M, M$ orais-Pinto NM, Pinto-Porto $C$, M oreira $T$, et al. $M$ etodologias ativas de ensino-aprendizagem na formação profissional em saúde: debates atuais. Ciênc Saúde Colet. 2008;13(2):2133-44.

10 A Imeida LPG, Ferraz CA. Políticas de formação de recursos humanos em saúde e enfer magem. Rev Bras Enferm. 2008;61(1):31-5.

11 Lucchese R, Vera I, Pereira W R. As políticas públicas de saúde - SU S - como referência para o processo ensino-aprendizagem do enfer meiro. Rev E letrônica E nferm [ I nternet] . 2010 [ citado $2010 \mathrm{dez} 12$ ] ;12(3): 562-6. Disponível em: http:/ / www.fen.ufg.br/ revis$\mathrm{ta} / \mathrm{v} 12 / \mathrm{n} 3 / \mathrm{v} 12 \mathrm{n} 3 \mathrm{a} 21 . \mathrm{htm}$.

12 M inistério da Saúde (BR), Secretaria de G estão do T rabalho e da E ducação em Saúde. A educação permanente entra na roda [I nternet]. Brasília (DF); 
2005 [ citado 2010 maio 20] . D isponível em: http:/ / dtr2001.saude.gov.br/ editora/ produtos/ livros/ pdf/ 05_0002_M 1.pdf.

Endereço da autora / Dirección del autor / Author's address:

Suzelaine T anji

Rua J oão Alves de M oura, 357

25964-190, Teresópolis, RJ

E-mail:jrdahmer@terra.com.br
Recebido em: 24/ 05/ 2010

A provado em: 23/ 08/ 2010 